\title{
Ionization potentials and radii of atoms and ions of element 104 (unnilquadium) and of hafnium $(2+)$ derived from multiconfiguration Dirac-Fock calculations
}

\author{
Elijah Johnson ${ }^{\text {a) }}$ and B. Fricke \\ Department of Physics, University of Kassel, D 3500 Kassel, West Germany \\ O. L. Keller, Jr., C. W. Nestor, Jr., ${ }^{\text {b) }}$ and T. C. Tucker') \\ Chemistry Division, Oak Ridge National Laboratory, Oak Ridge, Tennessee 37831-6375
}

(Received 11 January 1990; accepted 15 August 1990)

\begin{abstract}
Multiconfiguration relativistic Dirac-Fock (MCDF) values have been computed for the first four ionization potentials (IPs) of element 104 (unnilquadium) and of the other group 4 elements ( $\mathrm{Ti}, \mathrm{Zr}$, and $\mathrm{Hf}$ ). Factors were calculated that allowed correction of the systematic errors between the MCDF IPs and the experimental IPs. Single "experimental" IPs evaluated in $\mathrm{eV}($ to $\pm 0.1 \mathrm{eV})$ for element 104 are: [ 104(0),6.5]; [104(1+),14.8]; [104(2+),23.8]; $[104(3+), 31.9]$. Multiple experimental IPs evaluated in eV for element 104 are: $[(0-2+), 21.2 \pm 0.2] ;[(0-3+), 45.1 \pm 0.2] ;[(0-4+), 76.8 \pm 0.3]$. Our MCDF results track 11 of the 12 experimental single IPs studied for group 4 atoms and ions. The exception is $\mathrm{Hf}(2+)$. We submit our calculated IP of $22.4 \pm 0.2 \mathrm{eV}$ as much more accurate than the value of $23.3 \mathrm{eV}$ derived from experiment.
\end{abstract}

\section{INTRODUCTION}

According to the normal continuation of the Periodic Table, element 104 is expected to be a group 4 element below $\mathrm{Ti}, \mathrm{Zr}$, and $\mathrm{Hf}$ with a ground state configuration of $6 d^{2} 7 s^{2}$. Early Dirac-Fock computations in the single configuration approximation ${ }^{1}$ indicated that indeed the ground state is $6 d^{2} 7 s^{2}$, but we now confirm the more recent multiconfiguration Dirac-Fock (MCDF) results of Glebov et al. ${ }^{2}$ which give the ground state as $6 d 7 s^{2} 7 p$.

The MCDF values for the first four ionization potentials (IPs) of element 104 are reported here for the first time. We use these MCDF ionization potentials (MCDF IPs) to evaluate "experimental" IPs for the atom and ions of element 104 and for the poorly measured $\mathrm{Hf}(2+)$. Radii are also calculated.

Our main objective is to evaluate experimental IPs for element 104 that are accurate enough to be used in predictions of chemical properties. Chemical predictions of this kind are generally based on thermodynamic approaches as outlined for the transactinides by Cunningham ${ }^{3}$ and by Keller and co-workers. ${ }^{4}$ Some quantities needed-such as IPs and atomic radii-will be inaccessible to experiment for many years to come. These quantities now must come from theory. Other quantities, such as heats of sublimation ${ }^{5}$ and ionic radii, ${ }^{6,7}$ can be determined by experiment.

Experimental work on transactinide elements is very difficult because of their short half-life (seconds or less) and very low production rates (one atom at a time) ${ }^{8}$ Yet crucial experiments have been performed at heavy ion accelerators in the U.S., Soviet Union, and Germany where these elements are produced. The chemistry of element 104 was first

\footnotetext{
"Permanent address: Chemistry Division, Oak Ridge National Laboratory, P.O. Box 2008, Oak Ridge, Tennessee 37831-6375.

"Permanent address: Computing and Telecommunications Division, Oak Ridge National Laboratory, P.O. Box 2008, Oak Ridge, Tennessee 37831.
}

successfully studied by Zvara and co-workers using a gas thermochromatography technique they developed. ${ }^{9}$ Zvara's studies, which used the isotope ${ }^{259} 104\left(T_{1 / 2}=3.4 \mathrm{~s}\right)$, showed that element 104 behaved like $\mathrm{HfCl}_{4}$. Later Silva and co-workers carried out aqueous separations ${ }^{10}$ and Hulet and co-workers ${ }^{11}$ studied chloride complexation of 104 by ion exchange techniques using ${ }^{261} 104\left(T_{1 / 2}=65 \mathrm{~s}\right)$. Recently, Zhuikov and co-workers have established a lower limit for the heat of sublimation of element 104 from its chromatographic behavior. ${ }^{12}$ The results of these later experiments confirm the results of Zvara et al. ${ }^{9}$ An extensive discussion of the chemistry and physics of element 104 has been given by Hyde et al. ${ }^{13}$ What we know about the chemistry of element 104 compared to most other elements is clearly very limited. Larger targets of ${ }^{254}$ Es should become available from the Oak Ridge production facilities in the future. Then much higher production rates of transactinide elements at heavy ion accelerators will allow the development of a much broader knowledge of the chemical properties of these elements. $^{8}$

\section{METHOD}

The MCDF computer program used is described by Desclaux. ${ }^{14}$ The general theory is presented, e.g., by Grant, ${ }^{15}$ and Grant and Quiney. ${ }^{16}$ The basis functions used are linear combinations of Slater determinants. Each basis state (a configuration state function ${ }^{17}$ ) is an eigenfunction of both the square and the $z$ component of the total angular momentum operators. The single particle wave functions which build up the Slater determinants are four component spinors where each component is a product of spherical harmonics and radial functions. The Breit interaction was included by perturbation. The two radial functions (large and small components ) are obtained by solving the Dirac-Fock equations in an iterative procedure together with the expansion coefficients of the configuration state functions such 
that the system is self-consistent. The first iteration was started from the eigenfunctions in the Thomas-Fermi potential.

The MCDF calculations include all possible configuration state functions which can be constructed from the relativistic $n s, n p_{1 / 2}, n p_{3 / 2},(n-1) d_{3 / 2}$, and $(n-1) d_{5 / 2}$ single particle wave functions for a specific total angular momentum, $z$ component of the total angular momentum, and parity. For convenience, the $z$ component was always set equal to the total angular momentum in the calculations. Table I lists the five possibilities employed for positive parity states for the neutral to $4+$ ions. Column 1 lists all combinations of the single particle wave functions in the nonrelativistic nomenclature for the MCDF calculations for the neutral group 4 element where four electrons are distributed in the valence orbitals; column 2 lists the singly ionized species with three active electrons, etc. The possible number of configuration states which can be constructed with the various total angular momenta and parities possible are presented at the end of each column. The $f$ shells are energetically near; but since they are filled, we treated them as part of the core. Also the inclusion of linear combinations with the partially unfilled $f$ shell would result in far too many configurations for an actual calculation. Table II is analogous to Table I, but for negative parity states.

\section{RESULTS AND DISCUSSION}

\section{A. MCDF ground state energies}

Table III presents the MCDF ground state energies for the group 4 elements with charges from 0 to $4+$. Also for each atom and ion the number of configurations entering the calculation, the angular momentum and parity of the ground state, and the dominant resultant electronic configurations with their weights are presented. In order to derive these ground state energies for the neutral atom and ions of element 104, calculations were made using both the positive and negative parity configurations in Tables I and II.

The procedure used to calculate an MCDF ground state can be illustrated by the example of element $104(2+)$, where we have the configuration state functions constructed from the single particle functions $7 s, 7 p$, and $6 d$ along with the core $[\mathrm{Rn}] 5 f^{14}$ configuration. The three possibilities considered for the positive parity ground state are $J=0+$ through $J=2+$. The total energy of the lowest $J=0+$ through $J=2+$ states are calculated to be -1050261.048 , -1050259.848 , and $-1050259.634 \mathrm{eV}$, respectively. Since the $J=0+$ state has the lowest energy, it is the MCDF positive parity ground state for $104(2+)$. A similar comparison of the MCDF energies of the possible negative parity states shows that they also are higher in energy than the $J=0+$ state. So the $J=0+$ state is the true MCDF ground state of the 104( $2+$ ) ion. The angular momentum eigenvalues and the dominant configurations in $\mathrm{Ta}$ ble III so determined for the atoms and ions of $\mathrm{Ti}, \mathrm{Zr}$, and $\mathrm{Hf}$ are all in agreement with the experimental results. ${ }^{18}$

As can be seen in Table III, three of the five MCDF ground state angular momenta for element 104 differ from its homologs. The reason for this difference in behavior arises from the larger relativistic effects in 104. These relativistic effects are manifested in more $s$ and $p_{1 / 2}$ electron char$\operatorname{acter}^{19,20}$ in 104 than in its lighter homologs. A comparison has been made earlier between element 103 and its lighter homologs. ${ }^{21}$ The MCDF ground state of element 103 was calculated to be a $7 s^{2} 7 p$ state instead of the $6 d 7 s^{2}$ state exhibited by the lighter homologs. Analogously, the MCDF ground state of the neutral atom of element 104 is a negative parity state with a total angular momentum eigenvalue equal to 2 . This is because a relativistically stabilized $7 p_{1 / 2}$ electron replaces one of the $6 d$ electrons in the expected $6 d^{2} 7 s^{2}$ configuration. The resulting configuration is $[\mathrm{Rn}] 5 f^{14} 6 d 7 s^{2} 7 p$ with a weight of 0.804 . The energy of this $J=2-$ ground state is $-1050280.059 \mathrm{eV}$. The state with electronic energy closest to the ground state for the neutral element 104 atom is a $2+$ state consisting primarily of the $[R n] 5 f^{14} 6 d^{2} 7 s^{2}$ configuration with a configuration weight of 0.883 . The energy of this $2+$ state is $-1050279.822 \mathrm{eV}$. Thus we calculate that the energy difference between the relativistically

TABLE I. Configurations in the nonrelativistic nomenclature used in MCDF calculations for positive parity states. $^{2}$

\begin{tabular}{llll}
\hline \hline \multicolumn{1}{c}{$(0)$} & \multicolumn{3}{c}{ Configuration for charge $(q):\left(\mathrm{CORE}^{\mathrm{b}}\right)$ plus } \\
$(1+)$ & $(2+)$ & $(4+)$ & $(4+)$ \\
\hline$(n-1) d^{2} n s^{2}$ & $(n-1) d n s^{2}$ & $(n-1) d^{2}$ & $n s$ \\
$(n-1) d^{3} n s$ & $(n-1) d^{2} n s$ & $(n-1) d n s$ & \\
$(n-1) d^{4}$ & $(n-1) d^{3}$ & $n s^{2}$ & \\
$n s^{2} n p^{2}$ & $(n-1) d n p^{2}$ & $n p^{2}$ & \\
$(n-1) d n s n p^{2}$ & $n s n p^{2}$ & & \\
$(n-1) d^{2} n p^{2}$ & & & \\
$n p^{4}$ & & & \\
$J=0 ; 29^{\mathrm{c}}$ & & & \\
$J=1 ; 45$ & $J=1 / 2 ; 13$ & $J=0 ; 5$ & $J=1 / 2 ; 1$ \\
$J=2 ; 70$ & $J=3 / 2 ; 21$ & $J=1 ; 3$ & \\
$J=3 ; 50$ & & $J=2 ; 7$ & \\
& & & \\
\hline \hline
\end{tabular}

$n$ is the principal quantum number $(4,5,6$, and 7 for Ti, $\mathrm{Zr}, \mathrm{Hf}$, and 104$)$

${ }^{\mathrm{b}} \mathrm{CORE}=\mathrm{Ar}$ for $\mathrm{Ti} ; \mathrm{Kr}$ for $\mathrm{Zr}$; $\mathrm{Xe}(4 f)^{14}$ for $\mathrm{Hf}$; $\mathrm{Rn}(5 f)^{14}$ for 104.

${ }^{c}$ The number of configurations included in the calculation follows the associated $J$ value. 
TABLE II. Configurations in the nonrelativistic nomenclature used in MCDF calculations for negative parity states. $^{2}$

\begin{tabular}{|c|c|c|c|c|}
\hline \multicolumn{5}{|c|}{ Configuration for charge $(q):\left(\mathrm{CORE}^{\mathrm{b}}\right)$ plus } \\
\hline$(0)$ & $(1+)$ & $(2+)$ & $(3+)$ & $(4+)^{c}$ \\
\hline $\begin{array}{l}(n-1) d^{2} n s n p \\
(n-1) d^{3} n p \\
(n-1) d n s^{2} n p \\
(n-1) d n p^{3} \\
n s n p^{3}\end{array}$ & $\begin{array}{l}(n-1) d^{2} n p \\
(n-1) d n s n p \\
n s^{2} n p \\
n p^{3}\end{array}$ & $\begin{array}{l}(n-1) d n p \\
n s n p\end{array}$ & $n p$ & \\
\hline $\begin{array}{l}J=0 ; 20^{\mathrm{d}} \\
J=1 ; 53 \\
J=2 ; 65\end{array}$ & $\begin{array}{l}J=1 / 2 ; 13 \\
J=3 / 2 ; 22\end{array}$ & $\begin{array}{l}J=0 ; 2 \\
J=1 ; 5 \\
J=2 ; 5\end{array}$ & $\begin{array}{l}J=1 / 2 ; 1 \\
J=3 / 2 ; 1\end{array}$ & $J=0 ; 1$ \\
\hline
\end{tabular}

" $n$ is the principal quantum number $(4,5,6$, and 7 for $\mathrm{Ti}, \mathrm{Zr}, \mathrm{Hf}$, and 104$)$.

${ }^{b} \mathrm{CORE}=\mathrm{Ar}$ for $\mathrm{Ti}$; $\mathrm{Kr}$ for $\mathrm{Zr}$; $\mathrm{Xe}(4 f)^{14}$ for $\mathrm{Hf} ; \mathrm{Rn}(5 f)^{14}$ for 104 .

${ }^{c}$ A negative parity state was calculated only for $(104)^{4+}$ with the configuration $\operatorname{Rn}(5 f)^{13} 6 d$.

${ }^{\circ}$ The number of configurations included in the calculations follows the associated $J$ value.

stabilized 2 - state and the expected $2+$ state is $0.24 \mathrm{eV}$, which is in good agreement with the value of $0.5 \pm 0.2 \mathrm{eV}$ given by Glebov and co-workers ${ }^{2}$ for a similar calculation.

Other examples of relativistic stabilization of $7 \mathrm{~s}$ orbitals are found in $104(2+)$ and $104(3+)$. Since its ground state is dominantly $7 s^{2}$ rather than the expected $6 d^{2}$, the lowest energy state of $104(2+)$ has total angular momentum $J=0+$ rather than the $J=2+$ of $\operatorname{Ti}(2+), \operatorname{Zr}(2+)$, and $\mathrm{Hf}(2+)$. Similarly, $104(3+)$ is $J=1 / 2+$ rather than $3 / 2+$ because it is $7 s$ rather than $6 d$.

The values in Table III refer to ground states. In Table IV, we present a few typical results from the many MCDF calculations we have performed for excited states (relative to the ground state.) These excited state results will be helpful in estimating the errors associated with our calculated IPs and also in understanding the extrapolation procedures we have used to evaluate experimental IPs for element 104.

\section{B. Experimental ionization potentials}

In a practical MCDF calculation, the principal approximation is that a finite basis set of wave functions must be substituted for the infinite basis set actually required for a complete MCDF calculation. This implies that only part of the electron correlation is taken into account. In the calculations reported here, the configurations of interest are very much alike for $\mathrm{Ti}, \mathrm{Zr}$, and $\mathrm{Hf}$. The $J$ values of the outer valence electron shells are the same, and in most cases their level structures are also very similar for the same ionization stages. As a consequence, the errors in the correlation energies introduced by the finite basis sets are very much alike, or they will be only a slowly varying function which reflects the actual physical differences between the homologs in the ana$\log$ states. In addition, the calculations are very accurate from a numerical point of view. All of these factors combined allow us to evaluate predictable numerical corrections for the constant or systematically varying error between the MCDF IPs and the experimental IPs for Ti, Zr, and $\mathrm{Hf}$. As a practical criterion, we take enough configurations in our MCDF calculations to arrive at a precision of a few tenths of an $\mathrm{eV}$ in the systematic error.
We developed two models to evaluate the correction factors: one for multiple and the other for single IPs. Both models I and II, which are explained in detail below, contain a set of correction factors (called $\beta$ 's), which take account of the variation in the errors in the MCDF IPs as a function of $Z$ in group 4. Model I also contains a second set of correction factors (called $\Gamma$ 's), which take account of the variation in the MCDF errors as a function of the charge $(q)$ on ions of the same element $(Z)$. The $\beta$ 's and $\Gamma$ 's then are the precision (unsystematic error) of the MCDF IPs as a function of $Z$ and of charge, respectively. Models I and II allow the $\beta$ 's and $\Gamma$ 's to be determined to within a few tenths of an $\mathrm{eV}$. The $\beta$ 's and $\Gamma$ 's were extrapolated to element 104 and used to calculate the 104 IPs. The extrapolation is justified since the physics and mathematics contained in the MCDF calculation is so similar for element 104 and its homologs. Most of the ground states of 104 and its ions are different, however, from those in $\mathrm{Hf}, \mathrm{Zr}$, and $\mathrm{Ti}$ because of relativistic effects as already discussed above. Consequently, it might seem that we must extrapolate from a given level in the lighter homologs to the same level in 104 and then add the deexcitation energy to the ground state from Table IV. For simplicity, we do not present the two step process here since the deexcitation energy is added in automatically when the extrapolation is made directly to the ground state. In addition to the results for element 104, models I and II allowed the derivation of an accurate value for the poorly known IP of $\mathrm{Hf}(2+)$.

Since the needed experimental IPs do not appear as a group in any current data compilation, we give them in Table $\mathrm{V}$ with the references. It should be noted that our reference to C. E. Moore ${ }^{18}$ is to NSRDS-NBS 34, not to NSRDSNBS $35 .{ }^{22}$ In spite of the later number, the values in \#35, which can differ substantially from those in $\# 34$, are from the older literature.

\section{Ionization potential of $\mathrm{Hf}(2+)$}

During the development of the models used for calculating accurate IPs from the MCDF IPs, it became apparent that the IP of $23.3 \mathrm{eV}$ reported for $\mathrm{Hf}^{2+}$ in Moore ${ }^{18}$ (from Klinkenberg and co-workers ${ }^{31}$ ) was the only one of the 12 
TABLE III. Calculated electronic ground states for Ti, Zr, Hf, and element 104 in the charge states 0 to $4+$.

\begin{tabular}{|c|c|c|c|c|c|}
\hline \multirow{2}{*}{$\begin{array}{l}\text { Element } \\
\mathrm{Ti}\end{array}$} & \multirow{2}{*}{$\begin{array}{c}\begin{array}{c}\text { No. of } \\
\text { Configurations }\end{array} \\
70\end{array}$} & \multirow{2}{*}{$\begin{array}{l}\begin{array}{l}\text { Angular } \\
\text { momentum } \\
\text { and parity }\end{array} \\
2+\end{array}$} & \multicolumn{2}{|c|}{$\begin{array}{l}\text { Dominant configuration } \\
\text { and weights }\end{array}$} & $\begin{array}{l}\text { Total energy } \\
(\mathrm{eV})\end{array}$ \\
\hline & & & $\begin{array}{l}{[\mathrm{Ar}]} \\
3 d^{2} 4 s^{2} \\
3 d^{2} 4 p^{2}\end{array}$ & $\begin{array}{r}91.3 \% \\
7.7 \%\end{array}$ & \\
\hline $\mathrm{Zr}$ & 70 & $2+$ & $\begin{array}{l}3 d^{2} 4 p^{2} \\
{[\mathrm{Kr}]} \\
4 d^{2} 5 s^{2} \\
4 d^{2} 5 p^{2}\end{array}$ & $\begin{array}{r}89.2 \% \\
5.9 \%\end{array}$ & $\begin{array}{r}-23196.881 \\
-97793.982\end{array}$ \\
\hline $\mathrm{Hf}$ & 70 & $2+$ & $\begin{array}{l}{[\mathrm{Xe}](4 f)^{14}} \\
5 d^{2} 6 s^{2} \\
5 d^{2} 6 p^{2}\end{array}$ & $\begin{array}{r}88.3 \% \\
5.2 \%\end{array}$ & -409909.922 \\
\hline 104 & 65 & $2-$ & $\begin{array}{l}{[\mathrm{Rn}](5 f)^{14}} \\
6 d 7 s^{2} 7 p \\
6 d^{2} 7 s 7 p \\
6 d^{3} 7 p\end{array}$ & $\begin{array}{r}80.4 \% \\
15.3 \% \\
2.9 \%\end{array}$ & -1050280.059 \\
\hline $\operatorname{Ti}(1+)$ & 21 & $3 / 2+$ & $\begin{array}{l}{[\mathrm{Ar}]} \\
3 d^{2} 4 s\end{array}$ & $99.9 \%$ & -23190.475 \\
\hline $\operatorname{Zr}(1+)$ & 21 & $3 / 2+$ & $\begin{array}{l}{[\mathrm{Kr}]} \\
4 d^{2} 5 s\end{array}$ & $99.9 \%$ & -97787.853 \\
\hline $\mathrm{Hf}(1+)$ & 21 & $3 / 2+$ & $\begin{array}{l}{[\mathrm{Xe}](4 f)^{14}} \\
5 d 6 s^{2} \\
5 d^{2} 6 s\end{array}$ & $\begin{array}{r}84.0 \% \\
9.6 \%\end{array}$ & -409903.827 \\
\hline $104(1+)$ & 21 & $3 / 2+$ & $\begin{array}{l}{[\operatorname{Rn}](5 f)^{14}} \\
6 d 7 s^{2} \\
6 d^{2} 7 s \\
6 d 7 p^{2}\end{array}$ & $\begin{array}{r}89.9 \% \\
4.7 \% \\
2.8 \%\end{array}$ & -1050274.522 \\
\hline $\operatorname{Ti}(2+)$ & 7 & $2+$ & $\begin{array}{l}{[\mathrm{Ar}]} \\
3 d^{2}\end{array}$ & $99.9 \%$ & -23177.533 \\
\hline $\operatorname{Zr}(2+)$ & 7 & $2+$ & $\begin{array}{l}{[\mathrm{Kr}]} \\
4 d^{2}\end{array}$ & $99.9 \%$ & -97775.588 \\
\hline $\mathrm{Hf}(2+)$ & 7 & $2+$ & {$[\mathrm{Xe}](4 f)^{14}$} & $95.6 \%$ & \\
\hline $104(2+)$ & 5 & $0+$ & $\begin{array}{l}5 d 6 s \\
{[\mathrm{Rn}](5 f)^{14}}\end{array}$ & $4.4 \%$ & -409890.008 \\
\hline & & & $\begin{array}{l}7 s^{2} \\
6 d^{2} \\
7 p^{2}\end{array}$ & $\begin{array}{r}94.8 \% \\
3.2 \% \\
2.0 \%\end{array}$ & -1050261.048 \\
\hline $\operatorname{Ti}(3+)$ & 1 & $3 / 2+$ & $\begin{array}{l}{[\mathrm{Ar}]} \\
3 d\end{array}$ & $100.0 \%$ & -23150.983 \\
\hline $\operatorname{Zr}(3+)$ & 1 & $3 / 2+$ & $\begin{array}{l}{[\mathrm{Kr}]} \\
4 d\end{array}$ & $100.0 \%$ & -97753.279 \\
\hline $\mathrm{Hf}(3+)$ & 1 & $3 / 2+$ & $\begin{array}{l}{[\mathrm{Xe}](4 f)^{i 4}} \\
5 d\end{array}$ & $100.0 \%$ & -409868.460 \\
\hline $104(3+)$ & 1 & $1 / 2+$ & $\begin{array}{l}{[\mathrm{Rn}](5 f)^{14}} \\
7 s\end{array}$ & $100.0 \%$ & -1050238.112 \\
\hline $\operatorname{Ti}(4+)$ & 1 & $0+$ & {$[\mathrm{Ar}]$} & $100.0 \%$ & -23108.749 \\
\hline $\operatorname{Zr}(4+)$ & 1 & $0+$ & {$[\mathrm{Kr}]$} & $100.0 \%$ & -97719.860 \\
\hline $\mathbf{H f}(4+)$ & 1 & $0+$ & {$[\mathrm{Xe}](4 f)^{14}$} & $100.0 \%$ & -409836.196 \\
\hline $104(4+)$ & 1 & $0+$ & {$[\mathrm{Rn}](5 f)^{14}$} & $100.0 \%$ & -1050207.525 \\
\hline
\end{tabular}

IPs studied that does not fit our pattern. The physics and mathematics of the MCDF calculations is the same for $\mathrm{Hf}^{2+}$ as for the 11 other group 4 atoms and ions used to calibrate our $\beta$ 's and $\Gamma$ 's, so we see no reason why it should not fit the pattern as well as the rest of its homologs. On the basis of our calculations, we suggest that the ambiguities and missing levels discussed by Klinkenberg and co-workers in their paper ${ }^{31}$ led them to derive an incorrect IP for $\mathbf{H f}^{2+}$. In order to derive the IPs for element 104, we need the correct IP for $\mathrm{Hf}^{2+}$, so that will be our first task.

The determination of $\operatorname{IP}[\mathrm{Hf}(2+)]$ is made with the aid of Table VI. In Table VI, column 1 is the change in charge state for the ionization of one to four electrons of $\mathrm{Ti}$,
$\mathrm{Zr}$, and $\mathrm{Hf}$ (and 104). The corresponding angular momenta and dominant initial and final electron configurations are given in column 2. The MCDF IPs, given in column 3, are calculated by subtracting the energy (Table III) of the ground state neutral atom of each element from the energy of the upper charge state. For example, the energy of the $J=2+$ ground state of $\mathrm{Hf}(0)(-409909.922 \mathrm{eV})$ is subtracted from the energy of the $J=2+$ state of $\mathrm{Hf}(2+)$ $(-409890.008 \mathrm{eV})$ to give $19.91 \mathrm{eV}$. The experimental multiple electron IPs are given in column 4 . They are obtained by addition of the best of the experimental single elec. tron IPs recorded in Table $\mathrm{V}$.

The quantity $\alpha$ in column 5 of Table VI is the experi- 
TABLE IV. Promotion energies of selected atoms and ions.

\begin{tabular}{llccc}
\hline \hline \multicolumn{1}{c}{$\begin{array}{c}\text { Atom or } \\
\text { ion }\end{array}$} & \multicolumn{1}{c}{$\begin{array}{c}\text { Transition between ground } \\
\text { state and excited } \\
\text { configurations }\end{array}$} & $\begin{array}{c}\text { MCDF } \\
\text { energy } \\
(\mathrm{eV})\end{array}$ & $\begin{array}{c}\text { Exp. } \\
\text { energy } \\
(\mathrm{eV})\end{array}$ & $\begin{array}{c}\text { Difference } \\
\text { (MCDF-Exp. }) \\
(\mathrm{eV})\end{array}$ \\
\hline $\operatorname{Ti}(2+)$ & $3 d^{2}(J=2+) \rightarrow 3 d^{2}(J=0+)$ & 1.67 & 1.31 & 0.36 \\
$\operatorname{Ti}(3+)$ & $3 d(J=3 / 2+) \rightarrow 4 s(J=1 / 2+)$ & 9.72 & 9.97 & -0.25 \\
$\operatorname{Zr}(0)$ & $4 d^{2} 5 s^{2}(J=2+) \rightarrow 4 d^{2} 5 s^{2}(J=1+)$ & 0.81 & 0.54 & 0.27 \\
$\operatorname{Zr}(1+)$ & $4 d^{2} 5 s(J=3 / 2+) \rightarrow 4 d^{2} 5 s(J=1 / 2+)$ & 0.99 & 0.71 & 0.28 \\
$\operatorname{Zr}(2+)$ & $4 d^{2}(J=2+) \rightarrow 4 d^{2}(J=0+)$ & 1.25 & 1.00 & 0.25 \\
$\operatorname{Zr}(3+)$ & $4 d(J=3 / 2+) \rightarrow 5 s(J=1 / 2+)$ & 4.61 & 4.74 & 0.13 \\
$\operatorname{Hf}(0)$ & $5 d^{2} 6 s^{2}(J=2+) \rightarrow 5 d^{2} 6 s^{2}(J=1+)$ & 0.89 & 0.82 & 0.25 \\
$\operatorname{Hf}(1+)$ & $5 d 6 s^{2}(J=3 / 2+) \rightarrow 5 d^{2} 6 s(J=1 / 2+)$ & 1.75 & 1.50 & \\
$104(0)$ & $6 d 7 s^{2} 7 p(J=2-) \rightarrow 6 d^{2} 7 s^{2}(J=2+)$ & 0.24 & & \\
$104(1+)$ & $6 d 7 s^{2}(J=3 / 2+) \rightarrow 6 d^{2} 7 s(J=1 / 2+)$ & 3.02 & & \\
$104(2+)$ & $7 s^{2}(J=0+) \rightarrow 6 d^{2}(J=4+)$ & 3.96 & & \\
$104(3+)$ & $7 s(J=1 / 2+) \rightarrow 6 d(J=3 / 2+)$ & 0.34 & & \\
\hline \hline
\end{tabular}

${ }^{\text {a Reference } 21 .}$

mental IP minus the MCDF IP. Hence $\alpha$ is the total error in the MCDF IP calculation. It is illustrated in Fig. 1 as the relatively constant distance between the (a) experimental and (b) MCDF curves for the (0-2+) change in state for the group 4 elements. As can be seen from column 5, $\alpha$ is of the order of about 0.5 to $0.8 \mathrm{eV}$ per charge state. This is the magnitude one would expect from an extensive many-configuration MCDF calculation of the type we have done. ${ }^{36}$ The $\alpha$ 's are large in magnitude because they contain the systematic error from two different atomic entities being involved in the calculation of an MCDF IP. In contrast, if one takes the difference between two configurations which are in the same atom or ion, as in an excited state calculation, the

TABLE V. Experimental single ionization potentials for group 4 elements.

\begin{tabular}{lllll}
\hline \hline $\begin{array}{l}\mathrm{IP} \text { of } \\
\mathrm{Z}(n+)\end{array}$ & $\begin{array}{l}\text { Exp. IP } \\
\left(\mathrm{cm}^{-1}\right)\end{array}$ & $\begin{array}{l}\text { Exp. IP } \\
(\mathrm{eV})^{\mathrm{a}}\end{array}$ & $\begin{array}{l}\text { Error } \\
(\mathrm{eV})\end{array}$ & Ref. \\
\hline $\mathrm{Ti}(0)$ & 55010 & 6.820 & 0.012 & 23 \\
$\mathrm{Zr}(0)$ & 53506.0 & 6.78 & 0.02 & 24 \\
& & 6.6339 & 0.0004 & 25 \\
$\mathrm{Hf}(0)$ & 55047.9 & 6.82507 & 0.07 & 24 \\
& 54700 & 6.78 & 0.0004 & 26 \\
& & 6.52 & 0.1 & 23 \\
$\mathrm{Ti}(1+)$ & 109494 & 13.5755 & 0.0025 & 24 \\
& 109500 & 13.58 & 0.12 & 23 \\
& & 13.6 & 0.5 & 28 \\
$\mathrm{Zr}(1+)$ & 105900 & 13.13 & & 18 \\
$\mathrm{Hf}(1+)$ & 120000 & 14.9 & 0.1 & 23 \\
$\mathrm{Ti}(2+)$ & 221735.6 & 27.4917 & 0.0002 & 29 \\
& & 27.5 & 0.5 & 28 \\
$\mathrm{Zr}(2+)$ & 186400 & 23.11 & 0.06 & 30 \\
& 185400 & 22.99 & & 18 \\
$\mathrm{Hf}(2+)$ & & 23.3 & “a few $0.1 \mathrm{~V}$, & 18,31 \\
$\mathrm{Ti}(3+)$ & 348973.3 & 43.2672 & 0.0002 & 32 \\
$\mathrm{Zr}(3+)$ & 277605.8 & 34.4187 & 0.0002 & 33 \\
$\mathrm{Hf}(3+)$ & 269150 & 33.37 & 0.02 & 34 \\
& 269835 & 33.5 & 0.1 & 35 \\
& 268500 & 33.3 & 0.1 & 18 \\
\hline \hline & & & & \\
\hline
\end{tabular}

$8065.5410 \mathrm{~cm}^{-1}=1 \mathrm{eV}$. systematic error is largely removed, and we are left with what is essentially an unsystematic error that averages about $0.2 \mathrm{eV}$ as can be seen in Table IV. We have developed models that remove the large systematic error from the IPs and leave us with only the small unsystematic error for use in extrapolations to unknown IPs.

The parameter $\beta$ in column 6 of Table VI is the difference in the accuracy of the MCDF calculation for a given multiple IP in going from one $Z$ to the next higher $Z$. For example, $\beta[\operatorname{Zr}(0-4+), \quad \operatorname{Ti}(0-4+)]$ $=\alpha[\operatorname{Zr}(0-4+)]-\alpha[\operatorname{Ti}(0-4+)]=3.17-3.02$ $=0.2 \mathrm{eV}$. The $\beta$ 's for the $(0-2+)$ change in state of the group 4 elements are plotted in Fig. 1 (curve c). Just as $\beta$ is the variation of the MCDF IP calculations as a function of $Z$ (with the charge held constant), so $\Gamma$ in column 7 is the variation of the MCDF IPs as a function of charge (for the same $Z)$. For example, $\Gamma[\operatorname{Ti}(0-4+), \operatorname{Ti}(0-3+)]$ $=3.02-1.99=1.0 \mathrm{eV}$. An illustration of how well the MCDF IPs track the experimental ones for $\mathrm{Zr}$ and its ions is given in Fig. 2. The small variation in the $\Gamma$ 's and $\beta$ 's for $\mathrm{Zr}$ are shown in curves $\mathrm{c}$ and $\mathrm{d}$.

The experimentally determined $\beta$ 's (Table VI) for Ti, $\mathrm{Zr}$, and $\mathrm{Hf}$ are found to fall into the range of $0.1-0.4 \mathrm{eV}$. The $\Gamma$ 's for a given change in charge state in Table VI are also seen to vary from each other or from a trend by only a few tenths of an $\mathrm{eV}$. The $\Gamma$ 's for $[(0-2+),(0-1+)]$ for Ti, $\mathrm{Zr}$, and $\mathrm{Hf}$ increase in the trend 0.6,0.9,1.1 as a function of $Z$. $\Gamma[\operatorname{Ti}(0-3+), \operatorname{Ti}(0-2+)]$ and $\Gamma[\operatorname{Zr}(0-3+)$, $\operatorname{Zr}(0-2+)]$ are equal to 0.9 and 0.8 , respectively, and the $\Gamma$ 's for the $[(0-4+),(0-3+)]$ changes in state for $\mathrm{Ti}$ and $\mathrm{Zr}$ are both equal to 1.0. As in the case of the excitation energies and the variation of the $\beta$ 's, the variation in the $\Gamma$ 's indicates an unsystematic error close to $0.2 \mathrm{eV}$ in our MCDF calculations. Accordingly we will assume in our calculation of $\operatorname{IP}[\mathrm{Hf}(0-3+)]$ that $\Gamma[\mathrm{Hf}(0-3+), \operatorname{Hf}(0-2+)]$ is $0.8 \pm 0.2$.

The experimental values for the IPs of $\mathrm{Hf}(0-3+)$ and $\mathrm{Hf}(0-4+)$ are not recorded in Table VI because we discovered, as noted above, that the IP of $\mathrm{Hf}(2+)$ of 23.3 
TABLE VI. Multiple ionization potentials for group 4 elements. The IPs, $\alpha$ 's, and $\beta$ 's, evaluated according to model I, are in parentheses.

\begin{tabular}{|c|c|c|c|c|c|c|}
\hline $\begin{array}{l}\text { Element } \\
\text { and } \\
\text { change in } \\
\text { state }\end{array}$ & $\begin{array}{c}\text { Transition between } \\
\text { configurations } \\
\text { (see Table III) }\end{array}$ & $\begin{array}{c}\text { MCDF IP } \\
(\mathrm{eV})\end{array}$ & $\begin{array}{c}\text { Exp. IP } \\
(\mathrm{eV})\end{array}$ & $\begin{array}{c}\alpha \\
(\mathrm{eV})\end{array}$ & $\begin{array}{c}\beta \\
(\mathrm{eV})\end{array}$ & $\underset{(\mathrm{eV})}{\Gamma}$ \\
\hline \multicolumn{7}{|l|}{$\mathrm{Ti}$} \\
\hline $0-4+$ & $d^{2} s^{2}(J=2+)-\operatorname{CORE}(J=0+)$ & 88.13 & 91.15 & 3.02 & & 1.0 \\
\hline $0-3+$ & $d^{2} s^{2}(J=2+)-d(J=3 / 2+)$ & 45.90 & 47.89 & 1.99 & & 0.9 \\
\hline $0-1+$ & $d^{2} s^{2}(J=2+)-d^{2} s(J=3 / 2+)$ & 6.41 & 6.82 & 0.41 & & \\
\hline \multicolumn{7}{|l|}{$\mathrm{Zr}$} \\
\hline $0-4+$ & $d^{2} s^{2}(J=2+)-\operatorname{CORE}(J=0+)$ & 74.12 & 77.29 & 3.17 & 0.2 & 1.0 \\
\hline $0-3+$ & $d^{2} s^{2}(J=2+)-d(J=3 / 2+)$ & 40.70 & 42.87 & 2.17 & 0.2 & 0.8 \\
\hline $0-2+$ & $d^{2} s^{2}(J=2+)-d^{2}(J=2+)$ & 18.39 & 19.76 & 1.37 & 0.3 & 0.9 \\
\hline $0-1+$ & $d^{2} s^{2}(J=2+)-d^{2} s(J=3 / 2+)$ & 6.13 & 6.63 & 0.50 & 0.1 & \\
\hline \multicolumn{7}{|c|}{ 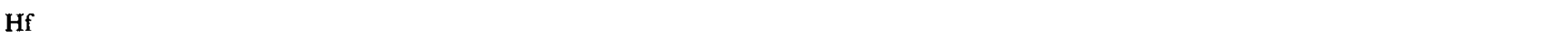 } \\
\hline $0-2+$ & $d^{2} s^{2}(J=2+)-d^{2}(J=2+)$ & 19.91 & 21.7 & 1.8 & 0.4 & 1.1 \\
\hline $0-1+$ & $d^{2} s^{2}(J=2+)-d s^{2}(J=3 / 2+)$ & 6.10 & 6.83 & 0.73 & 0.2 & \\
\hline \multicolumn{7}{|l|}{104} \\
\hline $0-4+$ & $d s^{2} p(J=2-)-\operatorname{CORE}(J=0+)$ & 72.53 & $(76.5)$ & $(4.0)$ & $(0.2)$ & $(1.0)$ \\
\hline $0-3+$ & $d s^{2} p(J=2-)-s(J=1 / 2+)$ & 41.95 & $(45.0)$ & $(3.0)$ & $(0.4)$ & $(0.8)$ \\
\hline $0-2+$ & $d s^{2} p(J=2-)-s^{2}(J=0+)$ & 19.01 & $(21.2)$ & $(2.2)$ & $(0.4)$ & $(1.2)$ \\
\hline $0-1+$ & (See Table VII) & & & & & \\
\hline
\end{tabular}
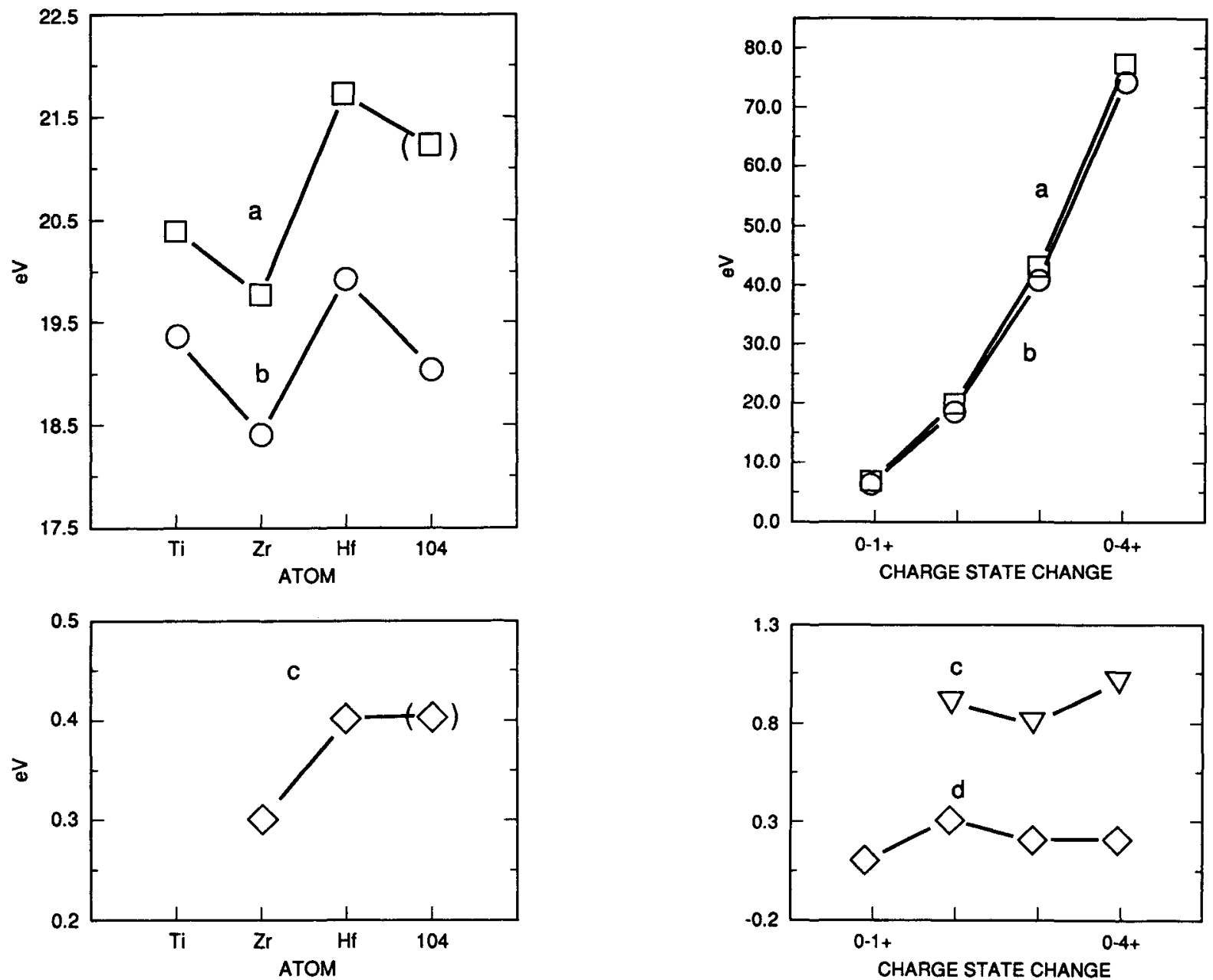

FIG. 1. (a) and (b) are experimental and MCDF IPs for the 0 to $2+$ change in state for group four elements and $(\mathrm{c})$ is the associated $\beta$. Element 104 values in parentheses are calculated from model I (see the text).

FIG. 2. (a) and (b) are experimental and MCDF IPs for the $(0-1+1$ through $(0-4+)$ changes in state for $Z r$. (c) is the associated $\Gamma$ and $(d)$ the associated $\beta$ (see the text). 
$\mathrm{eV}$ appearing in the Moore tables ${ }^{18}$ is inaccurate. The values for IP $[\mathrm{Hf}(0-3+)]$ and $\operatorname{IP}[\mathrm{Hf}(0-4+)]$ in Table VI are in parentheses to flag that they were evaluated using model I as described below.

Model $I$ is defined by specifying ranges that the $\beta$ 's and $\Gamma$ 's can assume in the calculation of the unknown IPs from the known IPs and the MCDF IPs. We will always let $\beta$ range from plus infinity to minus infinity for the change in state whose IP we are calculating. From this infinite set of $\beta$ 's, we will select only those IPs that yield $\Gamma$ 's in the range we have stipulated as described above. All IPs that yield $\Gamma$ 's outside the range will be discarded. It will be found that the $\beta$ 's selected by model I from the infinite set of $\beta$ 's are close to those found experimentally in Table VI. This approach of selecting the value of each $\beta$ according to the criterion set for the associated $\Gamma$ rather than specifying the $\beta$ 's as well as the $\Gamma$ 's directly serves as a check on the validity of model $I$.

As a first example, we specify the criterion that $\Gamma[\mathrm{Hf}(0-3+), \mathrm{Hf}(0-2+)]=0.8 \pm 0.2 \mathrm{eV}$ (as justified above) and then apply the above rules for $\beta$ and $\Gamma$ to evaluate $\operatorname{IP}[\mathrm{Hf}(0-3+)]$. The following steps were taken:

(1) For convenience, define a range variable, $\beta_{j}[\mathrm{Hf}(0-3+), \operatorname{Zr}(0-3+)]$ that runs from $0.0-0.7$ in steps of 0.1 . [It could run from $-\infty$ to $+\infty$, but the actual possible range for the $\beta_{j}$ 's under criterion (3) below will be found to be less than the chosen range of $0.0-0.7$.]

(2) Add each $\beta_{j}$ to $\alpha[\operatorname{Zr}(0-3+)](=2.17)$ to obtain a set of $\alpha_{j}[\mathrm{Hf}(0-3+)$ ]'s.

(3) Define $\Gamma_{j}[\mathbf{H f}(0-3+), \mathbf{H f}(0-2+)]$ $=\left\{\alpha_{j}[\mathrm{Hf}(0-3+)]-1.8\right\}$, where 1.8 is the experimentally known $\alpha[\mathrm{Hf}(0-2+)]$.

(4) Calculate each (IP) $)_{j}$ that satisfies the criterion $0.6 \leqslant \Gamma_{j}[\mathrm{Hf}(0-3+), \mathrm{Hf}(0-2+)] \leqslant 1.0$.

(5) Calculate the mean IP and the standard deviation. The method of extrapolation we have developed and applied here has never appeared in the literature to our knowledge. Only $\beta$ 's with values of 0.3 to 0.6 result in IPs that meet the criterion for the $\Gamma_{j}$ 's [item (4) above]. The associated mean value of IP $[\mathrm{Hf}(0-3+)]$ is $44.1 \pm 0.1 \mathrm{eV}$. Since the experimental $^{34} \operatorname{IP}[\mathrm{Hf}(3+)]=33.37, \operatorname{IP}[\mathrm{Hf}(0-4+)]=44.1$ $+33.37=77.5 \pm 0.1 \quad$ eV. Also $\quad \operatorname{IP}[\mathrm{Hf}(2+)]$ $=(44.1 \pm 0.1)-(21.7 \pm 0.1)=22.4 \pm 0.2 \mathrm{eV}$, where 21.7 is the experimental IP of $\mathrm{Hf}\left(\mathrm{O}_{-}+\right)$. These values, along with the $\beta$ and $\Gamma$ values derived from them, are given in Table VI in parentheses. The calculated mean $\beta$ selected from the infinite set by our calculation is 0.4 , a value that fits in with the experimental values.

As a further check on model I, IP $[\mathrm{Hf}(0-4+)]$ was calculated independently of $\operatorname{IP}\left[\mathrm{Hf}\left(\mathrm{O}_{-3}+\right)\right]$ and the experimental value of $\mathrm{Hf}(3+)$. The experimentally determined $\Gamma$ 's for the $[(0-4+),(0-3+)]$ change in state of $\mathrm{Ti}$ and $\mathrm{Zr}$ are both equal to 1.0. As discussed above, our MCDF unsystematic error is about $0.2 \mathrm{eV}$, so we assume that $\Gamma\left[\mathrm{Hf}\left(\mathrm{O}_{-}\right.\right.$ $4+), \mathrm{Hf}(0-3+)]$ is equal to $1.0 \pm 0.2 \mathrm{eV}$ for the model $\mathrm{I}$ calculation. The calculation using this criterion shows that only IPs associated with values of $\beta[\mathrm{Hf}(0-4+), \mathrm{Zr}(0-$ $4+$ )] from 0.1 through 0.8 are allowed. The mean $\mathrm{IP}=77.4 \pm 0.2 \mathrm{eV}$ thus calculated is in excellent agreement with the value of 77.5 calculated above from IP [ Hf $(0$ $3+)]$ and the experimental value of $\operatorname{IP}[\mathrm{Hf}(3+)]$ from Sugar. ${ }^{34}$

It will be found in the discussion of Table VII that our evaluated experimental IP of $22.4 \mathrm{eV}$ for $\mathrm{Hf}(2+)$ also yields a reasonable value for the $\beta$ for single IPs appropriate for that table. The fact that the value for the IP of $\mathrm{Hf}(2+)$ resulting from the multiple ionization calculation is consistent with the single ionization table also serves as a check on the model I calculation.

\section{Ionization potentials of element 104}

For the evaluations of the multiple IPs of element 104, we will use the same method (model I type calculation) employed to obtain IP $[\mathrm{Hf}(0-3+)]$ and $\operatorname{IP}[\mathrm{Hf}(0-4+)]$. The

TABLE VII. Single ionization potentials for group 4 elements. The IPs, $\alpha$ 's, and $\beta$ 's, evaluated according to model II, are in parentheses.

\begin{tabular}{|c|c|c|c|c|c|c|}
\hline $\begin{array}{l}\text { Change in } \\
\text { state }\end{array}$ & $\begin{array}{l}\text { Element } \\
(q)\end{array}$ & $\begin{array}{l}\text { Transition between } \\
\text { configurations } \\
\text { (see Table III) }\end{array}$ & $\begin{array}{l}\text { MCDF IP } \\
(\mathrm{eV})\end{array}$ & $\begin{array}{l}\text { Exp. IP } \\
(\mathrm{eV})\end{array}$ & $\begin{array}{l}\alpha \\
(\mathrm{eV})\end{array}$ & $\begin{array}{l}\beta \\
(\mathrm{eV})\end{array}$ \\
\hline \multirow[t]{4}{*}{$(0)-(1+)$} & $\operatorname{Ti}(0)$ & $d^{2} s^{2}(J=2+)-d^{2} s(J=3 / 2+)$ & 6.41 & 6.82 & 0.41 & \\
\hline & $\operatorname{Zr}(0)$ & $d^{2} s^{2}(J=2+)-d^{2} s(J=3 / 2+)$ & 6.13 & 6.63 & 0.50 & 0.1 \\
\hline & Hf $(0)$ & $d^{2} s^{2}(J=2+)-d s^{2}(J=3 / 2+)$ & 6.10 & 6.82 & 0.72 & 0.2 \\
\hline & $104(0)$ & $d s^{2} p(J=2-)-d s^{2}(J=3 / 2+)$ & 5.54 & $(6.5)$ & $(1.0)$ & $(0.3)$ \\
\hline \multirow{3}{*}{$(1+)-(2+)$} & $\operatorname{Zr}(1+)$ & $d^{2} s(J=3 / 2+)-d^{2}(J=2+)$ & 12.26 & 13.13 & 0.87 & 0.2 \\
\hline & $\mathrm{Hf}(1+)$ & $d s^{2}(J=3 / 2+)-d^{2}(J=2+)$ & 13.82 & 14.9 & 1.1 & 0.2 \\
\hline & $104(1+)$ & $d s^{2}(J=3 / 2+)-s^{2}(J=0+)$ & 13.47 & $(14.8)$ & $(1.3)$ & $(0.2)$ \\
\hline \multirow[t]{4}{*}{$(2+)-(3+)$} & $\operatorname{Ti}(2+)$ & $d^{2}(J=2+)-d(J=3 / 2+)$ & 26.55 & 27.49 & 0.94 & \\
\hline & $\operatorname{Zr}(2+)$ & $d^{2}(J=2+)-d(J=3 / 2+)$ & 22.31 & 23.11 & 0.80 & -0.1 \\
\hline & $\operatorname{Hf}(2+)$ & $d^{2}(J=2+)-d(J=3 / 2+)$ & 21.55 & $(22.4)$ & $(0.8)$ & $(0.0)$ \\
\hline & $104(2+)$ & $s^{2}(J=0+)-s(J=1 / 2+)$ & 22.94 & $(23.8)$ & $(0.9)$ & $(+0.1)$ \\
\hline \multirow[t]{3}{*}{$(3+)-(4+)$} & $\operatorname{Ti}(3+)$ & $d(J=3 / 2+)-\operatorname{CORE}(J=0+)$ & 42.23 & 43.27 & 1.04 & \\
\hline & $\mathrm{Hf}(3+)$ & $d(J=3 / 2+)-\operatorname{CORE}(J=0+)$ & 32.26 & 33.37 & 1.11 & 0.1 \\
\hline & $104(3+)$ & $s(J=1 / 2+)-\operatorname{CORE}(J=0+)$ & 30.59 & $(31.9)$ & $(1.3)$ & $(0.2)$ \\
\hline
\end{tabular}



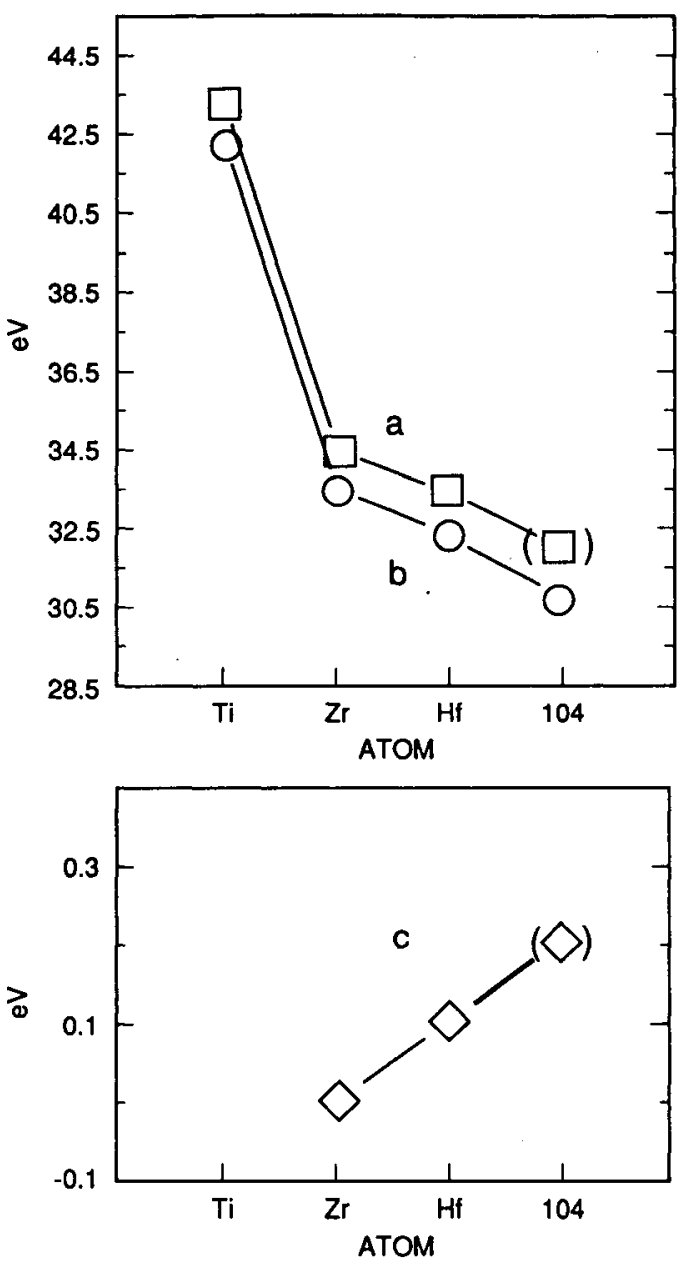

FIG. 3. (a) and (b) are experimental and MCDF IPs for the $3+$ ions of group 4 elements and $(\mathrm{c})$ is the associated $\beta$. Element 104 values in parentheses are calculated from model I (see the text).

$\Gamma$ ranges for the $[(0-3+),(0-2+)]$ and $[(0-4+),(0-$ $3+)$ ] changes in state of element 104 are taken to be the same as for the Hf calculations: $\{0.6 \leqslant \Gamma[104(0-3+)$; $104(0-2+)] \leqslant 1.0\} ; \quad$ and $\quad\{0.8 \leqslant \Gamma[104(0-4+)$, $104(0-3+)] \leqslant 1.2\}$. We will also need to set a range for $\Gamma$ for the $[(0-2+),(0-1+)]$ change in state of 104. In Table $V I$, the sequence for $\Gamma$ 's for the $(0-2+)$ change in state is $0.6(\mathrm{Ti}), 0.9(\mathrm{Zr})$, and $1.1(\mathrm{Hf})$. A continuation of the series to 104 gives the probable range $\{1.1 \leqslant \Gamma[104(0-$ $2+), 104(0-1+)] \leqslant 1.3\}$. The calculations for IP $[104(0-$
$3+)]$ and $\operatorname{IP}[104(0-4+)]$ closely parallel those of $\mathrm{Hf}(0$ $3+)]$ and $\mathrm{Hf}(0-4+)$. The experimental multiple IPs for element 104 thus evaluated with model $\mathbf{I}$ are: $(0-$ $2+)=21.2 \pm 0.1 ; \quad(0-3+)=45.0 \pm 0.2 ; \quad$ and $\quad(0-$ $4+)=76.5 \pm 0.2$. It should be noted that the mean $\beta$ 's selected by model I cause the calculated $\alpha$ 's and $\Gamma$ 's to fit perfectly in Table VI as though they had been calculated with Table VI rather than by the method that allows them to range from minus infinity to plus infinity. Also, as we shall see, calculations using another model based on single rather than multiple IPs will yield closely similar results. The final IPs for element 104 will be obtained by averaging the results of the two models.

We will now formulate model II based on the single MCDF IPs. This model is embedded in Table VII, which is for the single IPs of the group 4 elements. In Table VII, column 1 is the change in charge state for the one electron IP of the element or ion in column 2. The corresponding initial and final electron configurations and angular momenta are given in column 3. The MCDF IPs, given in column 4, are calculated by subtracting the energy (Table III) of the lower charge state from the upper. The experimental single IPs given in column 5 are the best values from Table V. The $\alpha$ 's and $\beta$ 's are defined for one electron changes analogously to those for the multiple IPs in Table VI. The excellent tracking of the experimental single IPs by the MCDF IPs is illustrat. ed in Fig. 3 for the $3+$ and $4+$ change in state. The ex. trapolations of the $\beta$ 's were so straightforward in Table VII that the $\Gamma$ 's were not needed for further differentiation. The following illustrates how Table VIII was set up: The $\beta[\operatorname{Zr}(0-1+), \operatorname{Ti}(0-1+)]=0.50-0.42=0.1$. It is re. corded in the $\operatorname{Zr}(0)$ line. Our calculated value of $22.4 \mathrm{eV}$ is used for the IP of $\mathrm{Hf}(2+)$ rather than the value of 23.3 derived from experiment (see the discussion above). It is seen that the $\beta=0.0$ for single electron changes resulting from the calculated value $\operatorname{IP}[\operatorname{Hf}(2+)]=22.4$ from model I fits nicely with the experimental value of $\mathrm{Zr}$, which equals -0.1 . This close fit supports the correctness of the calcular. tion of $\operatorname{IP}[\mathrm{Hf}(2+)]$ using model I.

The $\beta$ 's in Table VII give a clear pattern. Although the extrapolations to element 104 that we selected appear to be the only reasonable ones, we assume that the $\beta$ 's can vary from this value by $\pm 0.1 \mathrm{eV}$. This is equivalent to assuming an error of $\pm 0.1 \mathrm{eV}$ in the associated IP.

A new set of multiple IPs for element 104 results from model II by adding the single IPs in Table VII. These ner

TABLE VIII. Evaluated ionization potentials for element 104.

\begin{tabular}{cccc}
\hline $\begin{array}{c}\text { Change in } \\
\text { state }\end{array}$ & $\begin{array}{c}\text { Model I } \\
\text { IP } \\
(\mathrm{eV})\end{array}$ & $\begin{array}{c}\text { Model II } \\
\text { IP }^{\mathrm{b}} \\
(\mathrm{eV})\end{array}$ & $\begin{array}{c}\text { Avg. } \\
\text { IP } \\
(\mathrm{eV})\end{array}$ \\
\hline $0-1+$ & $\ldots$ & $6.5 \pm 0.1$ & $\ldots$ \\
$0-2+$ & $21.2 \pm 0.1$ & $21.3 \pm 0.2$ & $21.2 \pm 0.2$ \\
$0-3+$ & $45.0 \pm 0.2$ & $45.1 \pm 0.3$ & $45.1 \pm 0.2$ \\
$0-4+$ & $76.5 \pm 0.2$ & $77.0 \pm 0.4$ & $76.8 \pm 0.3$ \\
\hline
\end{tabular}

Table VI

${ }^{b}$ The sum of single IPs in Table VII. 
TABLE IX. The radius in nanometers of maximum radial charge density $\left(R_{\max }\right)$ in the outermost shell (shown in parentheses) of group 4 elements.

\begin{tabular}{cccccc}
\hline \hline Charge $\backslash$ Element & 0 & $1+$ & $2+$ & $3+$ & $4+$ \\
\hline $\mathrm{Ti}$ & $0.161(4 s)$ & $0.150(4 s)$ & $0.0523(3 d)$ & $0.0494(3 d)$ & $0.0469(3 p)$ \\
$\mathrm{Zr}$ & $0.173(5 s)$ & $0.163(5 s)$ & $0.0834(4 d)$ & $0.0798(4 d)$ & $0.0620(4 p)$ \\
$\mathrm{Hf}$ & $0.161(6 s)$ & $0.149(6 s)$ & $0.0872(5 d)$ & $0.0831(5 d)$ & $0.0626(5 p)$ \\
104 & $0.178(7 p)$ & $0.145(7 s)$ & $0.138(7 s)$ & $0.135(7 s)$ & $0.0706(6 p)$ \\
\hline
\end{tabular}

multiple IPs are compared in Table VIII to those calculated by model I using the multiple IPs approach of Table VI. Also in Table VIII the final IPs for element 104 are obtained as an average of the values from models I and II.

Table IX presents the position of the principal maxima $R_{\max }$ in the radial charge density functions of the occupied orbitals that have the largest values of $R_{\max }$ and that have a significantly large configuration weight associated with it for the group 4 elements. The values are taken for the electronic ground state configurations. As shown by Slater, the values of $R_{\max }$ are useful for estimating atomic and ionic radii. ${ }^{37,38}$

\section{CONCLUSION}

In all cases considered, the relativistic multiconfiguration Dirac-Fock (MCDF) total angular momentum eigenvalues and dominant configuration calculated for the lowest energy state agree with the experimental results. This indicates that the MCDF results are reliable at least for these two properties. Also MCDF calculations of excited state energies were found to be within about $0.2 \mathrm{eV}$ of the experimental values, an indication that unsystematic errors can be reduced to an acceptable minimum by taking a large enough basis set of wave functions (out of the theoretically required infinite set). An extrapolation method based on MCDF IPs has been developed and applied to obtain chemically useful IPs for $\mathrm{Hf}(2+)$ and the atom and ions through the $4+$ of element 104 . The extrapolation procedure necessarily depends on the availability of accurate IPs for the lighter homologs in group 4 of element 104. All IPs were available except for $\mathrm{Hf}(2+)$, which we believe to be inaccurately measured. We calculated a new value for the IP of $\mathrm{Hf}(2+)$. The MCDF ground state electronic configuration found for the neutral element 104 atom is different from that given by early predictions, ${ }^{1}$ but it is in agreement with that found by another recent MCDF calculation by Glebov et al. ${ }^{2}$

\section{ACKNOWLEDGMENTS}

Research sponsored by the Division of Chemical Sciences, Office of Basic Energy Sciences, U.S. Department of Energy under contract DE-AC05-84OR21400 with Martin Marietta Energy Systems, Inc., the Deutsche Forschungsgemeinschaft (DFG), and the Gesellschaft fur Schwerionenforschung (GSI), Darmstadt. The work was supported by the Florida State University Supercomputer Computations Research Institute, which is partially funded by the U.S.
Department of Energy through contract DE-FC05-85ER25000.

'B. Fricke, Struct. Bond. 21, 89 (1975).

${ }^{2}$ V. A. Glebov, L. Kasztura, V. S. Nefedov, and B. L. Zhuikov, Radiochim. Acta 46, 117 (1989).

${ }^{3}$ B. B. Cunningham, in Proceedings of the Robert A. Welch Foundation Conferences on Chemical Research XIII, The Transuranium Elements, edited by W. O. Milligan (R. A. Welch Foundation, Houston, 1970), Chap. XI.

${ }^{4}$ O. L. Keller, Jr., C. W. Nestor, and B. Fricke, J. Phys. Chem. 78, 1945 (1974).

${ }^{5}$ S. Hubener and I. Zvara, Radiochim. Acta. 31, 89 (1982).

${ }^{6}$ R. J. Silva, W. J. McDowell, O. L. Keller, Jr., and J. R. Tarrant, Inorg. Chem. 13, 2233 (1974)

${ }^{7}$ W. Bruchle, M. Schadel, U. W. Scherer, J. V. Kratz, K. E. Gregorich, D. Lee, M. Nurmia, R. M. Chasterler, H. L. Hall, R. A. Henderson, and D. C. Hoffman, Inorg. Chim. Acta. 146, 267 (1988).

${ }^{8}$ O. L. Keller, Radiochim. Acta. 37, 169 (1984).

${ }^{9}$ I. Zvara, V. Z. Belov, L. P. Chelnokov, V. P. Domanov, M. Hussonois, Yu. S. Korotkin, V. A. Schegolev, and M. R. Shalayevski, Inorg. Nucl. Chem. Lett. 7, 1109 (1971).

${ }^{10}$ R. Silva, J. Harris, M. Nurmia, K. Eskola, and A. Ghiorso, Inorg. Nucl. Chem. Lett. 6, 871 (1970).

${ }^{11}$ E. K. Hulet, R. W. Lougheed, J. F. Wild, J. H. Landrum, J. M. Nitschke, and A. Ghiroso, J. Inorg. Nucl. Chem. 42, 79 (1980).

${ }^{12}$ B. L. Zhuikov, Yu. T. Chuburkov, S. N. Timokhin, K. U. Jin, and I. Zvara, Radiochim. Acta 46, 113 (1989).

${ }^{13}$ E. K. Hyde, D. C. Hoffman, and O. L. Keller, Jr., Radiochim. Acta 42, 57 (1987).

${ }^{14}$ J.-P. Desclaux, Comput. Phys. Commun. 9, 31 (1975).

${ }^{15}$ I. P. Grant, Adv. Phys. 19, 747 (1970).

${ }^{16}$ I. P. Grant and H. M. Quiney, Adv. At. Mol. Phys. 23, 37 (1988).

${ }^{17}$ C. Froese Fischer, The Hartree-Fock Method for Atoms (Wiley, New York, 1977).

${ }^{18}$ Charlotte E. Moore, Ionization Potentials and Ionization Limits Derived from the Analyses of Optical Spectra, NSRDS-NBS 34, SD Catalog No. C 13.48:34 (Natl. Stand. Ref. Data Ser., Natl. Bur. Stand., Washington, D.C., 1970).

${ }^{19}$ K. S. Pitzer, Acc. Chem. Res. 12, 271 (1979).

${ }^{20}$ P. Pyykko and J.-P. Desclaux, Acc. Chem. Res. 12, 276 (1979).

${ }^{21}$ J.-P. Desclaux and B. Fricke, J. Phys. 41, 943 (1980).

${ }^{22}$ Charlotte E. Moore, Atomic Energy Levels, NSRDS-NBS 35, SD Catalog No. C 13.48:35, (Nat1. Stand. Ref. Data Ser., Natl. Bur. Stand., Washington, D.C., 1971).

${ }^{23}$ JANAF Thermochemical Tables, $3^{\text {rd }}$ ed., edited by M. W. Chase, C. A Davies, J. R. Downey, D. J. Frurip, R. A. McDonald, and A. N. Syverud (Natl. Bur. Stand., Washington, D.C., 1985).

${ }^{24}$ E. G. Rauh and R. J. Ackermann, J. Chem. Phys. 70, 1004 (1979).

${ }^{25}$ P. A. Hackett, M. R. Humphries, S. A. Mitchell, and D. M. Rayner, J. Chem. Phys. 85, 3194 (1986)

${ }^{26}$ C. L. Callender, P. A. Hackett, and D. M. Rayner, J. Opt. Soc. Am. B 5, 1341 (1988).

${ }^{27}$ S. Huldt, S. Johansson, and U. Litzen, Phys. Scr. 25, 401 (1982).

${ }^{28}$ M. J. Diserens, A. C. H. Smith, and M. F. A. Harrison, J. Phys. B: At. Mol. Opt. Phys. 21, 2129 (1988).

${ }^{29}$ B. Edlen and J. W. Swensson, Phys. Scr. 12, 21 (1975).

${ }^{30}$ Z. A. Khan, M. S. Z. Chaghtai, and K. Rahimullah, Phys. Scr. 23, 29 (1981).

${ }^{31}$ P. F. A. Klinkenberg, Th. A. M. Van Kleef, and P. E. Noorman, Physica 
27, 1177 (1961).

32 J. W. Swensson and B. Edlen, Phys. Scr. 9, 335 (1974).

${ }^{33}$ N. Acquista and J. Reader, J. Opt. Soc. Am. 70, 789 (1980).

${ }^{34}$ J. Sugar and V. Kaufman, J. Opt. Soc. Am. 64, 1656 (1974).

${ }^{35}$ F. G. Meijer, Physica 72, 431 (1974).
${ }^{36}$ K. Rashid, B. Fricke, J. H. Blanke, and J.-P. Desclaux, Z. Phys. D 11, 99 (1989).

${ }^{37}$ J. C. Slater, Quantum Theory of Molecules and Solids (McGraw-Hill, New York, 1965), Vol. 2, Chapter 4.

${ }^{38}$ J. C. Slater, J. Chem. Phys. 41, 3199 (1964). 